\title{
The Impact of Infective Immigrants on the Spread and Dynamics of Zika Viruss
}

\author{
Molalegn Ayana, Purnachandra Rao Koya ${ }^{*}$ \\ School of Mathematical and Statistical Sciences, Hawassa University, Hawassa, Ethiopia \\ Email address: \\ Molaayana@gmail.com (M. Ayana),drkpraocecc@yahoo.co.in (P. R. Koya) \\ ${ }^{*}$ Corresponding author
}

To cite this article:

Molalegn Ayana, Purnachandra Rao Koya. The Impact of Infective Immigrants on the Spread and Dynamics of Zika Viruss. American Journal of Applied Mathematics. Vol. 5, No. 6, 2017, pp. 145-153. doi: 10.11648/j.ajam.20170506.11

Received: May 9, 2017; Accepted: May 27, 2017; Published: November 5, 2017

\begin{abstract}
In this paper, a compartment model has been built, presented and investigated the dynamics and spread of zika virus in both human and mosquito populations. It is focused to study the impact of symptomatic and asymptomatic infective immigrants on the spread of zika virus. A new mathematical model $S I_{1} I_{2} R$ for human and $S I$ model for vector population has been designed and presented. Here $I_{1}$ is symptomatic infective and $I_{2}$ is asymptomatic infective human populations. The present model is developed making some reasonable modifications in the corresponding epidemic SIR model by considering symptomatic and asymptomatic infective immigrants. Susceptible vectors get infection either from symptomatic or asymptomatic infected human populations. The basic reproduction number is derived using the next generation matrix method. Disease free equilibrium point is found and endemic equilibrium state is identified. It is shown that the disease free equilibrium point is locally and globally asymptotically stable if the reproduction number takes a value less than one unit and unstable if it is more than one unit. Simulation study is conducted using MATLAB ode45.
\end{abstract}

Keywords: $S I_{1} I_{2} R$ Model, Symptomatic Infected, Asymptomatic Infected, Zika Virus, Microcephally

\section{Introduction}

Zika is a viral infection that is usually spread in human population by the bite of an infected mosquito. Zika was first discovered in 1947 in Uganda [12]. During the period $1960-$ 80 , human infections, typically accompanied by mild illness, were found across Africa and Asia. The first large outbreak of disease causing Zika infection occurred in the Island of Yap, Federated States of Micronesia in 2007, indicating that the virus had moved from South - East Asia across the Pacific. During 2013 - 14 outbreaks in French Polynesia the neurological disorder of Guillain-Barré syndrome or $G B S$ was found to have links to Zika infection. In South America, initially it was reported that the locally transmitted infection came from Brazil in 2015. In 2015 Brazil reported an association between Zika virus infection and $G B S$ disease.

From 1 April 2015, to 31March 2016 a total of 164,374 confirmed and suspected cases of Zika virus disease and 1474 cases of the GBS were reported in Bahia, Brazil, Colombia, the Dominican Republic, El Salvador, Honduras, Suriname, and Venezuela [16]. More cases of suspected
Microcephally temporally linked with Zika outbreak [14]. In February 2016, as infection moved rapidly through the range occupied by Aedes mosquitoes in the Americas, WHO declared that Zika infection associated with Microcephally and other neurological disorders constitutes a Public Health Emergency of International Concern (PHEIC).

By the start of 2016, local transmission of Zika infection had been reported from more than 20 countries and territories in the Americas, and an outbreak numbering thousands of cases was under way in Cabo Verde, western Africa. Approximately 500 million people in Latin America and the Caribbean are at risk for Zika virus infection [16].

Aedes aegypti mosquitoes live in tropical, subtropical, and in some temperate climates. These mosquitoes live near and prefer to feed on people. They are considered highly efficient at spreading these diseases.

There is no evidence that Zika spreads through touching, coughing, or sneezing. There is no vaccine or specific treatment for Zika virus infection. The most common 
symptoms of Zika are fever, rash, joint pain, conjunctivitis or red eyes, muscle pain, and headache. Patients zika virus infected are advised to drink plenty of fluids to replenish fluid lost from sweating, vomiting and other insensible losses [13].

Many communities along with national agencies initiated the process to implement control measures that ranged from vector control and the use of repellents to the suggestion of avoiding pregnancies for two years [3]. In addition to Microcephaly the other problems found in pregnancies, among fetuses and infants infected with Zika virus before birth such as: miscarriage, stillbirth, absent or poorly developed brain structures, defects of the eyes, hearing deficits, and impaired growths [3].

Due to association between Zika virus infection and Microcephaly, the epidemic trajectory of this viral infection poses a significant concern for nearly 15 million children born in Americas each year [6].

The impact of migration of population on the distribution and spread of zika virus disease is required to be analyzed properly and must be understood clearly. Migration and immigration of the people from one country to another country due to different reasons play a crucial role in the evolution and spread of zika virus.

The main objective of the present study is to understand the role of infective immigrants on the dynamics of zika virus, and also to develop effective solutions and strategies for its prevention and control and eventually to eradicate it.

Symptomatic infected human: The symptomatic infective humans are those who have already been infected by the disease and they show the symptoms of the disease and can transfer the disease to susceptible vector.

Asymptomatic infected human: The asymptotic infective humans have already infected by the disease but they do not show any symptoms of the disease. However, they can transfer the disease to susceptible vector. It has been estimated that about $80 \%$ of persons infected with zika virus are asymptomatic [3].

\section{Mathematical Formulation of the Model}

The mathematical model is expected to help to understand better the transmission and spread of zika. The spread of the disease modeled using ordinary differential equations ODEs where humans and mosquitoes interact and infect each other.

For the purpose of this study the whole human population is classified in to four compartments: Susceptible $S(t)$, Symptomatic infected $I_{1}(t)$, Asymptomatic infected $I_{2}(t)$, and Recovered $R(t)$. Similarly, the mosquito population is classified into two compartments: susceptible, and infected.

Many mathematical models did not consider symptomatic and asymptomatic infected immigrants on the dynamics of zika virus $[1-3]$. To fill the gap and to account its impact the present study considers it. Thus, a new model that combines a $S I_{1} I_{2} R$ system for disease transmission in the human hosts and $S I$ model for disease transmission in the mosquito vector has been constructed. The variables used in the model and their physical interpretations are as follows:

Table 1. The model variables and their representations.

\begin{tabular}{ll}
\hline$S_{h}(t)$ & Number of susceptible human population at any time t \\
$I_{1}(t)$ & Number of Symptomatic infected human population at any time $\mathrm{t}$ \\
$I_{2}(t)$ & Number of asymptomatic infected human population at any time $\mathrm{t}$ \\
$R_{h}(t)$ & Number of recovered human population at any time $\mathrm{t}$ \\
$S_{v}(t)$ & Number of susceptible vector population at any time $\mathrm{t}$ \\
$I_{v}(t)$ & Infected vector population at any time $\mathrm{t}$ \\
\hline
\end{tabular}

The vector component of the model does not include an immune class as mosquitoes never recover from the infection. That is, their infective period ends with their death due to their relatively short lifecycle. Thus, the immune class in the mosquito population is negligible and death occurs equally in all classes.

The model can be used for diseases that persist in a population for a long period of time.

Susceptible human compartment: People will join the susceptible compartment by natural birth or by immigration. People go out from this compartment by natural death or go to the symptomatic or asymptomatic infected classes. The remaining people will stay in the compartment itself. Peoples in the susceptible compartment will get infection from infected vector.

Infected human compartments: Into infected compartment some people will enter from susceptible after getting infection. Some others will enter by immigrations from other places. Some people of the infected human compartment will die with natural reasons or go to recovered class after developing immunity.

Recovered compartment: Human populations in recovered class will go out of the compartment through natural death.

Susceptible vector compartment: Vectors will join the susceptible class by natural birth and leaves the compartment through natural death. They go to the infected class after getting infection from symptomatic or asymptomatic infected humans.

Infected vector compartment: The infected vector leaves the compartment through natural death because of short life cycle, but not of infection.

In these model $\Lambda_{h} N_{h}$ and $\Lambda_{v} N_{v}$ represents the total recruitment rate of human and mosquito population respectively.

The susceptible human population get infection by the rate $b \Phi$, where $\Phi$ is the rate at which susceptible human becomes infected because of infected vector and $b$ is the biting rate of infected mosquito at which susceptible human becomes infected because of infected vector. The symptomatic infected immigrants come to the symptomatic infected human population by the rate $P_{1}$ and the asymptomatic infected immigrants come to the asymptomatic infected human population by the rate $P_{2}$. The symptomatic and asymptomatic infected human population goes to the recovered class equally by the rate $\delta$ and from the compartment due to natural death by the rate $\mu_{h} I_{1}$ and $\mu_{h} I_{2}$ respectively. The recovered human leave the compartment due to natural death by the rate $\mu_{h} R_{h}$. The susceptible 
mosquito population get infection either from symptomatic or asymptomatic infected humans by the rate $\theta$ and respectively, and leave the compartment due to natural death by the rate $\mu_{v} S_{v}$ and goes to infected mosquito compartment. Note that in this model we consider $>\theta$. That is, the probability of transferring the disease to susceptible vector by asymptomatic infected person is more than by symptomatic infected person. The infected mosquito population infects the susceptible human population by the rate $\Phi$ and leaves the compartment due to natural death by the rate $\mu_{v} I_{v}$.

Table 2. Description of variables and parameters.

\begin{tabular}{|c|c|}
\hline$\Lambda_{h}$ & Recruitment rate of human population \\
\hline$\mu_{h}$ & Natural death rate of human population \\
\hline$b$ & Biting rate of infected mosquito \\
\hline$\Phi$ & Rate of infection of susceptible human because of infected vector \\
\hline$P_{1}$ & Symptomatic infected human immigration rate \\
\hline$P_{2}$ & Asymptomatic infected human immigration rate \\
\hline$\delta$ & $\begin{array}{l}\text { Recovered rate of symptomatic and asymptomatic infected } \\
\text { human population }\end{array}$ \\
\hline$\Lambda_{v}$ & Recruitment rate of mosquito population \\
\hline$\mu_{v}$ & Natural death rate of mosquito population \\
\hline$\theta$ & $\begin{array}{l}\text { Rate at which susceptible vector get infection symptomatic } \\
\text { infected human }\end{array}$ \\
\hline & $\begin{array}{l}\text { Rate at which susceptible vector get infection from } \\
\text { asymptomatic infected human }\end{array}$ \\
\hline$\varepsilon$ & Rate at which susceptible human goes to symptomatic infected \\
\hline $1-\varepsilon$ & $\begin{array}{l}\text { Rate at which susceptible human becomes asymptomatic } \\
\text { infected }\end{array}$ \\
\hline
\end{tabular}

\subsection{Model Assumptions}

The present model has been developed based on the following assumptions:

(i) Susceptible vectors can be infected by symptomatic or asymptomatic infected humans.

(ii) Infected mosquitoes cannot recovery from the infection. Infected mosquitoes die naturally but not due to disease or infection and the birth rate of mosquito equals with its death rate..

(iii) Infected humans do not die due to infection, but due to natural death.

(iv) All the new born ones are susceptible to infection.

(v) The model considers only mosquito as a transmission agent. That is, infected mosquito can infect a susceptible human; but, infected human cannot infect a susceptible human.

(vi) The development of the virus starts when the infected mosquito bites the human host.

(vii) The infection is propagated to vectors by asymptomatic infected hosts with more rates than by symptomatic infected hosts.

(viii) The recovered individuals are assumed to acquire permanent immunity and there is no transfer from the $R$ class back to the $S$ class.

(ix) Susceptible vectors can get infection, through direct contact with a symptomatic or asymptomatic infected individual.

(x) Natural death or due to other diseases occurs in each of the compartments.

(xi) Populations move from one compartment to another compartment with certain rates.

Based on these assumptions the compartmental structure of the model showing the compartments and flow of population has been represented by a flow diagram as follows:

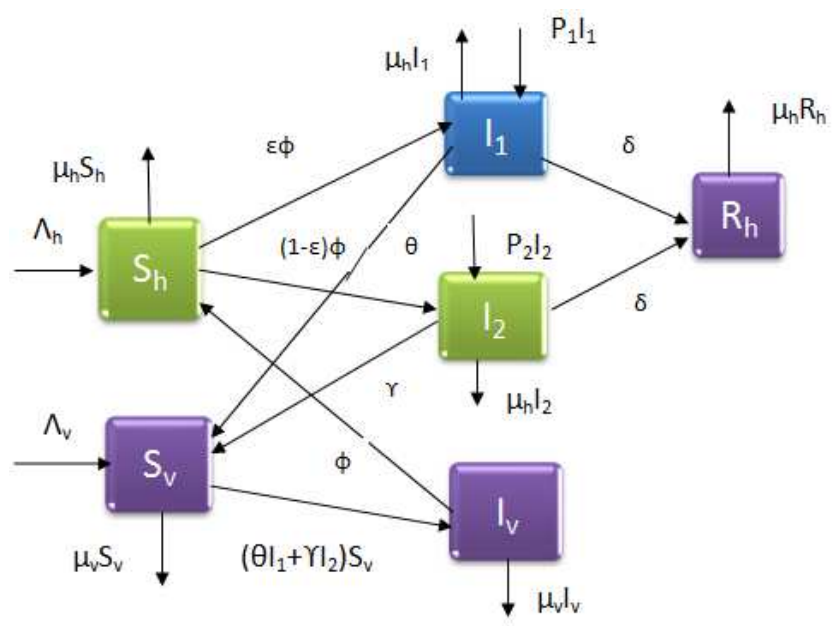

Figure 1. The compartmental structure describing Zika virus transmission.

Considering the model assumptions and the flow diagram given in Figure 1 the dynamics of zika virus in human and mosquito populations can be described by a system of ordinary differential equations constructed in terms of notations of variables and parameters as follows:

$$
\begin{gathered}
d S_{h} / d t=\Lambda_{h} N_{h}-b \emptyset I_{v}\left(S_{h} / N_{h}\right)-\mu_{h} S_{h} \\
d I_{1} / d t=b \in \emptyset I_{v}\left(S_{h} / N_{h}\right)+p_{1} I_{1}-\left(\mu_{h}+\delta\right) I_{1} \\
d I_{2} / d t=b(1-\in) \emptyset I_{v}\left(S_{h} / N_{h}\right)+p_{2} I_{2}-\left(\mu_{h}+\delta\right) I_{2} \\
d R_{H} / d t=\delta\left(I_{1}+I_{2}\right)-\mu_{h} R_{H} \\
d S_{V} / d t=\Lambda_{V} N_{V}-b \theta I_{1}\left(S_{V} / N_{h}\right)-b \gamma I_{2}\left(S_{V} / N_{h}\right)-\mu_{V} S_{V} \\
d I_{V} / d t=b \theta I_{1}\left(S_{V} / N_{h}\right)+b \gamma I_{2}\left(S_{V} / N_{h}\right)-\mu_{V} I_{V}
\end{gathered}
$$

With initial conditions $S_{h}(0)=S_{h 0}, I_{1}(0)=I_{10}, I_{2}(0)=$ $I_{20}$,

$$
R_{h}(0)=R_{h 0}, S_{v}(0)=S_{v 0}, I_{v}(0)=I_{v 0}
$$

The total population sizes $N_{h}$ and $N_{v}$ can be determined by

$$
N_{h}=S_{h}+I_{1}+I_{2}+R_{h} \text { and } N_{v}=S_{v}+I_{v}
$$

\subsection{Positivity of the Solution}

The general model equations of system (1-6) are to be epidemiologically meaningful and well posed; we need to prove that all the state variables are non-negative.

Theorem 1: If $s_{h}(0)>0, i_{1}(0)>0,, i_{2}(0)>0$, $i_{v}(0)$ then the solutions $\left\{S_{h}(t), I_{1}(t), I_{2}(t), R_{h}(t), S_{v}(t), i_{v}(t)\right\}$ on the system of equation (1-6) are non-negative for all $t>0$.

Proof: To show the positivity of the solution of the dynamical system comprising the equations (1) to (6) we have to consider and verify each differential equation and 
show that their solution is positive.

First let us consider equation (1) of the dynamical system

$$
\begin{gathered}
d S_{h} / d t=\Lambda_{h} N_{h}-b \emptyset I_{v}\left(S_{h} / N_{h}\right)-\mu_{h} S_{h}, \\
d S_{h} / d t \geq-b \emptyset I_{v}\left(S_{h} / N_{h}\right)-\mu_{h} S_{h}, d S_{h} / d t \geq-\lambda S_{h}- \\
\mu_{h} S_{h} \geq-\left(\lambda+\mu_{h}\right) S_{h}
\end{gathered}
$$

Where $\lambda=b \emptyset\left(I_{v} / N_{h}\right)$. Up on integrating the inequality we obtain analytic solution as $S_{h}(t) \geq S_{h 0} e^{\int-\left(\lambda+\mu_{h}\right) d t}$. Here $S_{h o}$ is integral constant and represents initial population of the susceptible human compartment and hence it is a positive quantity. Now within the limit $t \rightarrow \infty$ the analytical solution leads to $S_{h}(t)>0$. Hence the solution or the population size of the susceptible compartment $S_{h}(t)$ is always positive.

Secondly let's consider equation (2) of the dynamical system $\quad d I_{1} / d t=b \in \emptyset I_{v}\left(S_{h} / N_{h}\right)+p_{1} I_{1}-\left(\mu_{h}+\delta\right) I_{1}$ which implies $d I_{1} / d t \geq b \in \emptyset I_{v}\left(S_{h} / N_{h}\right)-k I_{1}$,where $k=\mu_{h}+\delta-p_{1} d I_{1} / d t \geq-k I_{1}$, Integrating by using separation of variables we have the first order linear ordinary differential equation and can be solved to obtain a particular solution as $I_{1}(t) \geq I_{10} e^{-k t}$, Since $I_{10}>0$, and the exponential function always positive, it is clear that $I_{1}(t) \geq$. Similarly $I_{2}(t) \geq I_{10} e^{-q t}$, where $q=\mu_{h}+\delta-p_{2}$.

Thirdly, let's consider equation (4) of the dynamical system $\quad d R_{h} / d t=\delta\left(I_{1}+I_{2}\right)-\mu_{h} R_{H}$ which can be expressed as $d R_{H} / d t \geq-\mu_{h} R_{H}$. Up on integrating the inequality we obtain an analytic solution as $R_{H}(t) \geq$ $R_{h 0} e^{-\mu_{h} t}$. Since $R_{h 0}>0$, and the exponential function always positive, it is clear that $R_{h}(t) \geq 0$.

Fourthly consider equation (5) of the dynamical system $d S_{V} / d t=\Lambda_{V} N_{V}-b \theta I_{1}\left(S_{V} / N_{h}\right)-b \gamma I_{2}\left(S_{V} / N_{h}\right)-$ $\mu_{V} S_{V}$ without the loss of generality this can be expressed as $d S_{V} / d t-r(t) S_{V}-\mu_{V} S_{V}$ where $(t)=b \theta\left(I_{1} / N_{h}\right)-$ $b \gamma\left(I_{2} / N_{h}\right)$. Integrating by using separation of variable we have the first order linear ordinary differential equation and can be solved to obtain a particular solution as $S_{V}(t)=$ $S_{V 0} e^{\int-r(t) d t-\mu_{V} t}$. Since $S_{V 0}>0$ and the exponential function always positive, it is clear that $S_{v}(t) \geq 0$.

Finally let's consider equation (6) of the model equation which is $d I_{V} / d t=b \theta I_{1}\left(S_{V} / N_{h}\right)+b \gamma I_{2}\left(S_{V} / N_{h}\right)-$ $\mu_{V} I_{V}$ without the loss of generality this can be expressed as $d I_{V} / d t \geq-\mu_{V} I_{V}$. Upon integrating the inequality we obtain the analytic solution as $I_{v}(t)=I_{v 0} e^{-\mu_{V} t}$. Here $I_{v 0}$ is integral constant and represent initial infective vector population and hence it is a positive quantity. Now within the limit $t \rightarrow \infty$ the analytical solution leads to $I_{v}(t) \geq 0$. Hence the solution or the population size of the infected compartment $I_{v}(t)$ is always non-negative.

Therefore the solution sets $\left\{S_{h}(t), I_{1}(t), I_{2}(t), R_{h}(t), S_{v}(t), I_{v}(t)\right\} \quad$ of the model equations (1) to (6) are all non-negative for all $t \geq 0$.

\subsection{Boundedness of the Solution}

The total human population $N_{h}(t)=S_{h}(t)+I_{1}(t)+$ $I_{2}(t)+R_{h}(t)$ implies using equations (1) to (4) $d N_{h} / d t=$ $d S_{h} / d t+d I_{1} / d t+d I_{2} / d t+d R_{h} / d t=\Lambda_{h} N_{h}-\mu_{h} N_{h}+$ $P_{1} I_{1}+P_{2} I_{2}$ (8) Here without loss of generality and physical meaning $I_{1}<N_{h}, I_{2}<N_{h}$ and $I_{1}+I_{2} \leq N_{h}$. which implies $d N_{h} / d t \leq \Lambda_{h} N_{h}-\mu_{h} N_{h}+P_{1} N_{h}+P_{2} N_{h}$ solving this we have the analytic solution of the form $N_{h}(t) \leq\left(\Lambda_{h}+P_{1}+\right.$ $\left.P_{2}\right) N_{h} / \mu_{h}-N_{0} e^{-\mu_{h}}$ or equivalently it implies that $0<$ $N_{h}(t) \leq\left(\Lambda_{h}+P_{1}+P_{2}\right) N_{h} / \mu_{h}$ as $t \rightarrow \infty$. Therefore the solution system of human population is bounded. Similarly the total vector population is $N_{v}(t)=S_{v}(t)+I_{v}(t)$ implies using equations (5) and (6) we have $d N_{v} / d t=d S_{v} / d t+$ $d I_{v} / d t=\Lambda_{v} N_{v}-\mu_{v} N_{v}$. Since in our assumption the natural birth rate and the death rate of vector is equal then, $\Lambda_{v}=\mu_{v}$ which implies the vector population is constant and equal with the initial vector population which is bounded. Therefore the feasible region is

$$
\begin{gathered}
\Omega=\Omega_{h} X \Omega_{v}=\left\{S_{h}, I_{1}, I_{2}, R_{h}, S_{v}, I_{v} \in R^{6}: N_{h}\right. \\
\left.\leq \frac{\left(\Lambda_{h}+P_{1}+P_{2}\right) N_{h}}{\mu_{h}}, N_{v}=N_{0}\right\}
\end{gathered}
$$

\subsection{Disease Free Equilibrium Point}

Disease-free equilibrium (DFE) points are steady-state solutions where there is no Zika virus infection i.e $I_{1}=I_{2}=$ $R_{h}=I_{v}=0$. This is obtained by setting the right hand side of the model equation equal to zero. Thus, the disease-free equilibrium point of the model equation is, $E_{0}=\left\{S_{h}, I_{1}, I_{2}, R_{h}, S_{v}, I_{v}\right\}=\left\{\Lambda_{h} N_{h} / \mu_{h}, 0,0,0, \Lambda_{v} N_{v} / \mu_{v}, 0\right\}$.

\subsection{Basic Reproduction Number}

The basic reproduction number denoted by $R_{0}$, is defined as the number of secondary infections that result from the introduction of a single infectious individual into a completely susceptible population during its entire period of infectiousness, will be calculated by using the next generation matrix technique. It is an important parameter in epidemiology as it sets the threshold in the study of a disease both for predicting its outbreak as well as evaluating its control strategies. Persistent and die out of the disease in a community depend on the size of the reproduction number, $R_{0}$. If the reproductions number $R_{0}>1$ the disease break out and if the reproduction number $R_{0}<1$, the disease dies out over the period of time. When $R_{0}=1$ then the disease becomes endemic, meaning the disease remains in the population at a constant rate as one infected human transmits the disease to one susceptible vector and one infected vector transmits the disease to one susceptible human.

Now by considering the infective compartments in human and mosquito population we can compute the reproduction number by using next generation approach. The infected compartments are $I_{1}, I_{2}, I_{v}$. From those infected compartments we can compute the infection state $F$ and transfer state $V$. The reproduction number is the dominant eigenvalue of the matrix $F V^{-1}$. The rate of change of population in this compartments are give in the dynamic model equation $(2,3)$ and equation(6). From this we can find $f_{i}$ and $v_{i}$. 


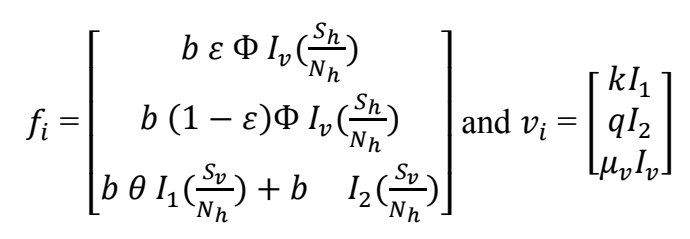

Differentiating with respect to the variables $I_{1}, I_{2}, I_{v}$, and solving at the disease free equilibrium point $E_{0}$ gives the jacobian matrix

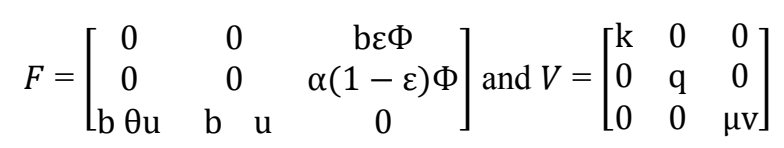

Here we have used the fact initially $S_{h}=N_{h}$. Finally after computing we have got the matrix

$$
F V^{-1}=\left[\begin{array}{ccc}
0 & 0 & \frac{\mathrm{b} \varepsilon \Phi}{\mu_{\mathrm{v}}} \\
0 & 0 & \frac{\mathrm{b}(1-\varepsilon) \Phi}{\mu_{\mathrm{v}}} \\
\frac{\mathrm{b} \theta \mathrm{u}}{\mathrm{k}} & \frac{\mathrm{b} \Upsilon \mathrm{u}}{\mathrm{q}} & 0
\end{array}\right] \text { where } u=m \frac{\Lambda_{v}}{\mu_{v}}, m=\frac{N_{v}}{N_{h}}
$$

The eigenvalues of the matrix $F V^{-1}$ are the solution of the characteristic equation $\left|F V^{-1}-\lambda I\right|=0$ where $I$ is an identity matrix and $\lambda$ is the Eigen value of the matrix $F V^{-1}$. Solving this we have $\lambda_{1}=0, \lambda_{2}=-\sqrt{\frac{b^{2} u \Phi}{\mu_{v}}\left(\frac{(1-\varepsilon)}{q}+\frac{\theta \varepsilon}{k}\right)}, \lambda_{3}=\sqrt{\frac{b^{2} u \Phi}{\mu_{v}}\left(\frac{(1-\varepsilon)}{q}+\frac{\theta \varepsilon}{k}\right)}$.

Among those eigenvalues the dominant Eigen value is $\lambda_{3}=\sqrt{\frac{b^{2} u \Phi}{\mu_{v}}\left(\frac{(1-\varepsilon)}{q}+\frac{\theta \varepsilon}{k}\right)}$ which is the reproduction number. i.e. $R_{0}=\sqrt{\frac{b^{2} u \Phi}{\mu_{v}}\left(\frac{(1-\varepsilon)}{q}+\frac{\theta \varepsilon}{k}\right)}$. The reproduction number is a powerful parameter which measures the existence and stability of the disease in the human and mosquito population.

\section{Analysis of the Model}

In this section, the equilibrium points for the general model are identified and their stability analysis is made. The system exhibits two types of equilibrium point viz., disease free equilibrium points and endemic equilibrium points.

\subsection{Scaling of the Model}

In order to simplify and analyze the zika model in system (1-6) we work with fractional quantities instead of actual populations by scaling the population of each class by the total species population as papers $[4,8,11]$. The model can be simplified by assuming the following fractions with conditions $S_{h}=s_{h} N_{h}, I_{1}=i_{1} N_{h}, I_{2}=i_{2} N_{h}, R_{h}=r_{h} N_{h}, S_{v}=$ $s_{v} N_{v}, I_{v}=i_{v} N_{v}$ therefore in the scaled version $s_{h}+i_{1}+$ $i_{2}+r_{h}=1$ and $s_{v}+i_{v}=1$. From this $s_{v}=1-i_{v}$, $r_{h}=1-s_{h}-i_{1}-i_{2}$. In our model since recovered individuals develop permanent immunity and hence they do not propagate the disease either to susceptible or to infected vector. That is $I_{1}$ and $I_{2}$ are not depend on $R_{h}$ therefore without loss of generality this system of equation can be written as subsystem of four equations. Doing by differentiating the fractions with respect to time $t$ and simplifying as follows and the scaled version of the model becomes

$$
\begin{gathered}
d s_{h} / d t=\Lambda-\alpha s_{h} i_{v}-\mu_{\mathrm{h}} \mathrm{s}_{\mathrm{h}} \\
d i_{1} / d t=\alpha \varepsilon i_{v} s_{h}-\mu_{h} i_{1}-\delta i_{1}+P_{1} i_{1} \\
d i_{2} / d t=\alpha(1-\varepsilon) i_{v} s_{h}-\mu_{h} i_{2}-\delta i_{2}+P_{2} i_{2} \\
d i_{v} / d t=\mathrm{b} \theta \mathrm{i}_{1} \mathrm{~s}_{\mathrm{v}}+\mathrm{b} \quad \mathrm{i}_{2} \mathrm{~s}_{\mathrm{v}}-\mu_{\mathrm{v}} \mathrm{i}_{\mathrm{v}}
\end{gathered}
$$

Where $\alpha=b \Phi m$

\subsection{Existence and Stability of Disease Free Equilibrium}

Disease-free equilibrium (DFE) points are steady-state solutions where there is no Zika virus infection and the equilibrium points are obtained by setting the right hand sides of the model equations to zero.

Theorem1: The disease free equilibrium point is locally asymptotically stable if and only if the reproduction number $R_{0}<1$ and unstable if $R_{0}>1$.

Proof: The local stability of $E_{0}$ is then determined from the signs of the eigenvalues of the Jacobian matrix. At the disease-free equilibrium, $E_{0}$, the Jacobian matrix is given by

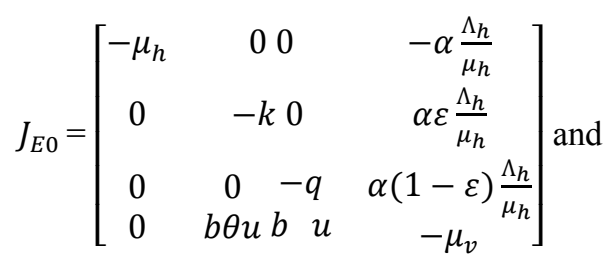

$$
\begin{aligned}
& \operatorname{det}\left(J_{E 0}-\lambda I\right)=\left|\begin{array}{cccc}
-(\mu h+\lambda) & 0 & 0 & -\alpha \eta \\
0 & -(k+\lambda) & 0 & \alpha \varepsilon \eta \\
0 & 0 & -(q+\lambda) & \alpha(1-\varepsilon) \eta \\
0 & b \theta u & b \Upsilon u & -(\mu v+\lambda)
\end{array}\right|
\end{aligned}
$$

Where, $\eta=\frac{\Lambda_{h}}{\mu_{h}}$ solving $Q(\lambda)=\operatorname{det}\left(J_{\mathrm{E} 0}-\lambda I\right)=0$ we have $-\left(\mu_{h}+\lambda\right)=0 \Rightarrow \lambda=-\mu_{h}$.

The other three Eigenvalues are the roots of the characteristic equation of the matrix formed by excluding the first row and first column of the Jacobean matrix and has the characteristic equation of the form

$$
\lambda^{3}+a_{1} \lambda_{2}+a_{2} \lambda+a_{3}=0
$$

Where,

$a_{1}=k+q+\mu_{v}>0, a \quad 2 \quad=k q+k \mu_{v}+$ $q \mu_{v}-\alpha b \eta u \Upsilon(1-\varepsilon)-\alpha b \eta u \theta \varepsilon$ and $a_{3}=k q \mu_{v}(1-$ $\left.R_{0}^{2}\right)>0$ if and only if $R_{0}<1$.

Due to the complexity in determining the signs of the remaining eigenvalues, we employ Routh-Hurwitz conditions for stability. The roots of the characteristic equation have negative real parts if and only if all the principal diagonal minors of the Hurwitz matrix are positive. For our case of a third order system, the stability criterion is defined by the inequalities

$a_{1}>0, a_{3}>0$ and $a_{1} a_{2}-a_{3}>0$. Clearly $a_{1}>0, a_{3}>$ 0 and $a_{2}$ to be positive 
$k q+k \mu_{v}+q \mu_{v}>\alpha b u \eta \Upsilon(1-\varepsilon)+\alpha b \eta u \theta \varepsilon \quad$ and also $a_{1} a_{2}-a_{3}>0$.

Hence, by Routh-Hurwitz criterion, all the eigenvalues have negative real parts if $R_{0}<1$, Hence from RouthHurwitz criterion we have all the real parts of eigenvalues of the Hurwitz matrix being negative, hence the disease free equilibrium point is stable if $R_{0}<1$.

Theorem 2: The disease free equilibrium point $E_{0}$ of the system of ordinary differential equations is globally stable if $R_{0}<1$.

Proof: The proof is based on using a comparison theorem. The equation of the infected components can be written in terms of

$$
\begin{gathered}
{\left[\begin{array}{l}
d I_{1} / d t \\
d I_{2} / d t \\
d I_{v} / d t
\end{array}\right]=(F-V)\left[\begin{array}{l}
I_{1} \\
I_{2} \\
I_{v}
\end{array}\right]-\left[\begin{array}{c}
0 \\
0 \\
\left(b \quad \theta I_{1}+b \Upsilon I_{2}\right) I_{v}
\end{array}\right]} \\
{\left[\begin{array}{l}
d I_{1} / d t \\
d I_{2} / d t \\
d I_{v} / d t
\end{array}\right] \leq(F-V)\left[\begin{array}{l}
I_{1} \\
I_{2} \\
I_{v}
\end{array}\right]}
\end{gathered}
$$

And also all the eigenvalues of the matrix $[F-V]$ have negative real parts. It follows that the system of linear differential inequalities (15) is stable whenever $R_{0}<1$ [19].

\subsection{The Endemic Equilibrium Point}

Endemic equilibrium point is a steady-state solution, where the disease persists in human and mosquito population. This equilibrium implies that if the carrier population is present in the system, then the infection will be transmitted to the human and mosquito population. Endemic equilibrium $E_{1}$ of our model is obtained by setting right hand side of the model system of equation equal to zero. For the existence and uniqueness of endemic equilibrium $E_{1}$ $=\left(S_{h}^{*}, I_{1}^{*}, I_{2}^{*}, I_{v}^{*}\right)$ where $=\left(S_{h}^{*}, I_{1}^{*}, I_{2}^{*}, I_{v}^{*}\right)$ and solving system above by setting the right hand side of the equation equals to zero we have the following $\mathrm{E}_{1}=\left(S_{h}^{*}, I_{1}^{*}, I_{2}^{*}, I_{v}^{*}\right)$

$$
\begin{aligned}
& S_{h}^{*}=\frac{\Lambda_{h}\left(r R_{0}^{2}+1\right)}{\mathrm{R}_{0}^{2} \mu_{\mathrm{h}}(r+1)} I_{1}^{*}=\frac{\varepsilon \Lambda_{h}\left(R_{0}^{2}-1\right)}{k R_{0}^{2}(r+1)} I_{2}^{*}=\frac{(1-\varepsilon) \Lambda_{h}\left(R_{0}^{2}-1\right)}{q R_{0}^{2}(r+1)} \\
& I_{v}^{*}=\frac{\alpha \mathrm{b} \Lambda_{\mathrm{h}} \mathrm{k} \Upsilon \mathrm{u}(1-\varepsilon)+\alpha \mathrm{b} \Lambda_{\mathrm{h}} \mathrm{qu} \theta \varepsilon-\mu_{\mathrm{v}} \mu_{h} \mathrm{kq}}{\alpha \mathrm{b} \Lambda_{\mathrm{h}} \mathrm{ku} \Upsilon(1-\varepsilon)+\alpha \mathrm{b} \Lambda_{\mathrm{h}} \mathrm{qu} \theta \varepsilon+\alpha \mu_{\mathrm{v}} \mathrm{kq}}=\frac{\mu_{\mathrm{h}}\left(\mathrm{R}_{0}^{2}-1\right)}{\alpha\left(\mathrm{r} \mathrm{R}_{0}^{2}+1\right)}, \\
& \text { where } r=\mu_{h} / \alpha
\end{aligned}
$$

Here $I_{v}^{*}$ is positive if and only if $R_{0}>1$ and therefore all endemic equilibrium points can be expressed in terms of $R_{0}$ and all are positive. We apply the linearization technique in the system to determine the stability of the endemic equilibrium.

Theorem 3:- The Endemic equilibrium point of system of equation is locally asymptotically stable if and only if the reproduction number $R_{0}>1$ and unstable if $R_{0}<1$.

Proof: At the steady states of the model, the Jacobean matrix is given by

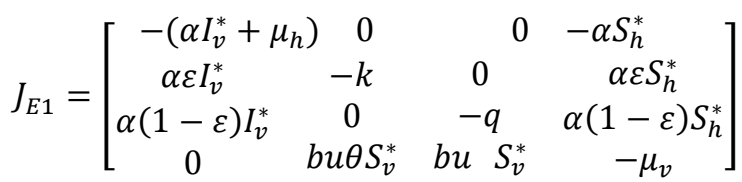

The characteristic polynomial at the endemic equilibrium point is given by $P(\lambda)=\operatorname{det}\left(J_{E 1}-\lambda I\right)=0$, and therefore

$$
P(\lambda)=\lambda^{4}+a_{1} \lambda^{3}+a_{2} \lambda^{2}+a_{3} \lambda+a_{4}=0,
$$

Where

$$
\begin{aligned}
& a_{1}=\alpha I_{v}^{*}+\mu_{h}+k+q \mu_{v}>0 \text { if and only if } R_{0}>1 \\
& \begin{aligned}
a_{2}=\alpha k I_{v}^{*}+ & \alpha q I_{v}^{*}+\alpha \mu_{v} I_{v}^{*}+\mu_{h} k+\mu_{h} q+\mu_{h} \mu_{v} \\
& +k q+k \mu_{v}+q \mu_{v} \\
& -\alpha b u \gamma(1-\varepsilon) S_{h}^{*} S_{v}^{*}-\alpha b \theta u \varepsilon S_{h}^{*} S_{v}^{*} .
\end{aligned}
\end{aligned}
$$

$$
\begin{aligned}
a_{3}=\alpha k q+ & \alpha k \mu_{v} I_{v}^{*}+\alpha q \mu_{v} I_{v}^{*}+\mu_{h} k q+\mu_{h} \mu_{v} k \\
& +\mu_{h} \mu_{v} q+\mu_{h} \alpha b u(1-\varepsilon) S_{h}^{*} S_{v}^{*} \\
& -\mu_{h} \alpha b u \theta \varepsilon S_{h}^{*} S_{v}^{*}+k q \mu_{v} \\
& -k \alpha b u \gamma(1-\varepsilon) S_{h}^{*} S_{v}^{*}-\alpha b q u \theta \varepsilon S_{h}^{*} S_{v}^{*}
\end{aligned}
$$

$a_{4}=\alpha k q \mu_{v} I_{v}^{*}+k q \mu_{v} \mu_{h}-\alpha b \mu_{h} k u \gamma(1-$ ع) $S_{h}^{*} S_{v}^{*}-\alpha b \mu_{h} q u \theta \varepsilon S_{h}^{*} S_{v}^{*}$. Since solving the above characteristic polynomial for eigenvalues is tedious we will use the Routh-Hurwitz criterion to determine whether all roots have negative real parts and establish the stability of the system without solving the characteristic equation itself. By Routh - Hurtwiz criteria the determinant of Hurwitz matrix becomes positive if the following conditions hold true $a_{1}>0, a_{3}>0$ and $a_{4}>0$ and $a_{1} a_{2} a_{3}>a_{1}^{2} a_{4}+a_{3}^{2}$. It required that all these requirements should hold true in our present model. Therefore, after a certain manipulation we have the following results

$a_{2}>0$ if and only if $R_{0}>1$ and $b \Lambda_{h} \mu_{h}\left\{\gamma(1-\varepsilon)\left(R_{0}^{2}+\right.\right.$ 1) $\left.+\theta \varepsilon\left(R_{0}^{2}-1\right)\right\}>\alpha b \Lambda_{h}\left(r R_{0}^{2}+1\right)\{u \gamma(1-\varepsilon)+u \theta \varepsilon\}$.

$a_{3}>0$ if and only if $R_{0}>1$ and $\Lambda_{h} b\left(R_{0}^{2}-1\right)\left\{\mu_{h} \theta \varepsilon+\right.$ $b \gamma u(1-\varepsilon)-q \theta \varepsilon\}>\alpha b \Lambda_{h}\left(r R_{0}^{2}+1\right)\{\theta u \varepsilon+$

$\left.\left(q u \theta \varepsilon / \mu_{h}\right)+k u \gamma(1-\varepsilon) / \mu_{h}\right\}$

$a 4>0$ iff $R 0>1$ and $b \Lambda_{h}\left(R_{0}^{2}-1\right)\{k u \gamma(1-\varepsilon)-$ $q u \theta\}>\alpha b \Lambda_{h}\left(r R_{0}^{2}+1\right)\{k u \gamma(1-\varepsilon)+q u \theta\}$

Hear all the requirements on the parameters are found hold well.

Similarly, after exercising the same procedure it can be further observed that $a_{1} a_{2}>a_{3}$ and $a_{1} a_{2} a_{3}>a_{1}^{2} a_{4}+a_{3}^{2}$ holds with a certain conditions. Hence all roots of the characteristic polynomial are negative and this verifies that the system of equation (1) to (6) is locally asymptotically stable.

\section{Simulation Study of the Model.}

Our numerical simulations examine the effect of infective symptomatic and asymptomatic immigrants on the transmission of the disease using MATLAB ode 45 and show the sensitive parameters on the dynamics of the disease. Some of the parameter values are taken from literatures and the others are by assumptions. The parametric values are given in the following table. 
Table 3. Model parameters and their values.

\begin{tabular}{lllll}
\hline Parameters & Case 1 & Case 2 & Case 3 & Reference \\
\hline$\Lambda_{h}$ & 0.06 & 0.06 & 0.06 & {$[17]$} \\
$\mu_{h}$ & 0.003 & 0.003 & 0.003 & {$[19]$} \\
$\mu_{v}$ & 0.029 & 0.029 & 0.029 & {$[11]$} \\
$b$ & 0.3 & 0.3 & 0.3 & {$[20]$} \\
$b \Phi$ & 0.75 & 0.65 & 0.4 & Assumption \\
$\theta$ & 0.25 & 0.63 & 0.1333 & Assumption \\
& 0.4 & 0.7 & 0.2 & Assumption \\
$\delta$ & 0.2 & 0.2 & 0.2 & {$[21]$} \\
$P_{1}$ & 0.1 & 0.2 & 0.1 & Assumption \\
$P_{2}$ & 0.2 & 0.3 & 0.1 & Assumption \\
$\varepsilon$ & 0.2 & 0.2 & 0.2 & Assumption \\
$\alpha$ & 0.4218 & 0.3656 & 0.225 & Assumption \\
\hline
\end{tabular}

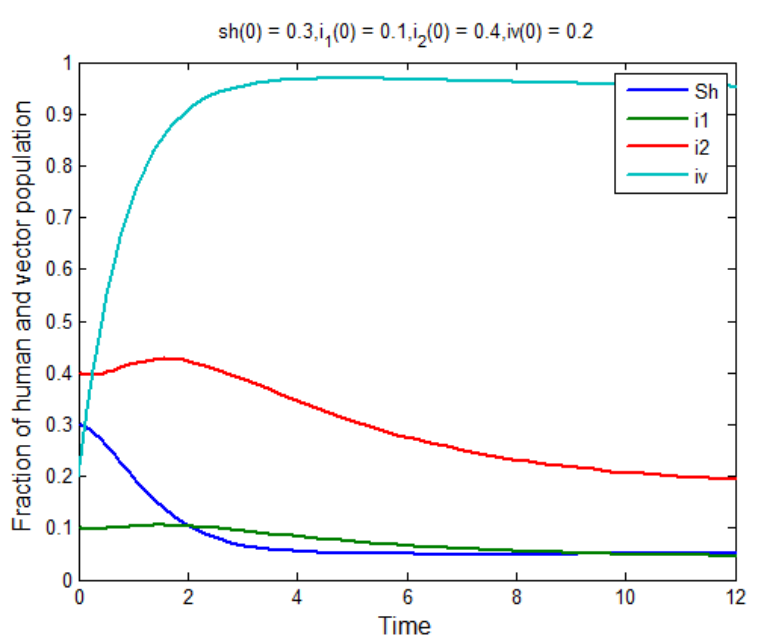

Figure 2. Numerical simulation of zika virus with $\theta=0.25, \quad=0.4, \alpha=$ $0.4218, P_{1}=0.1, P_{2}=0.2$.

In figure 2 the fractions of the populations $s_{h}, i_{1}, i_{2}$, and $i_{v}$ are plotted vs. time. With increasing time, the susceptible fraction of human population and symptomatic infected human populations are decrease and the fractions of infected vector populations increase.

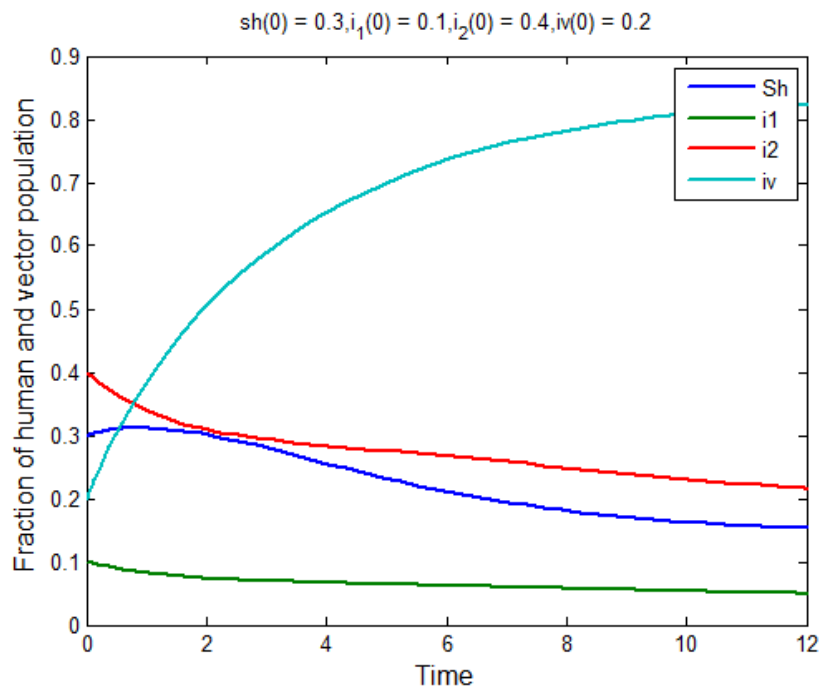

Figure 3. Numerical simulation of zika virus with $\theta=0.63, \quad=0.7, \alpha=$ $0.365, P_{1}=0.2, P_{2}=0.3$.

In figure 3 the parameters are changed and with increasing time, the fractions of susceptible humans and the fraction of asymptomatic infected humans decrease and infected vectors are increases very fast because of the values of $P_{2}$ and $\Upsilon$.

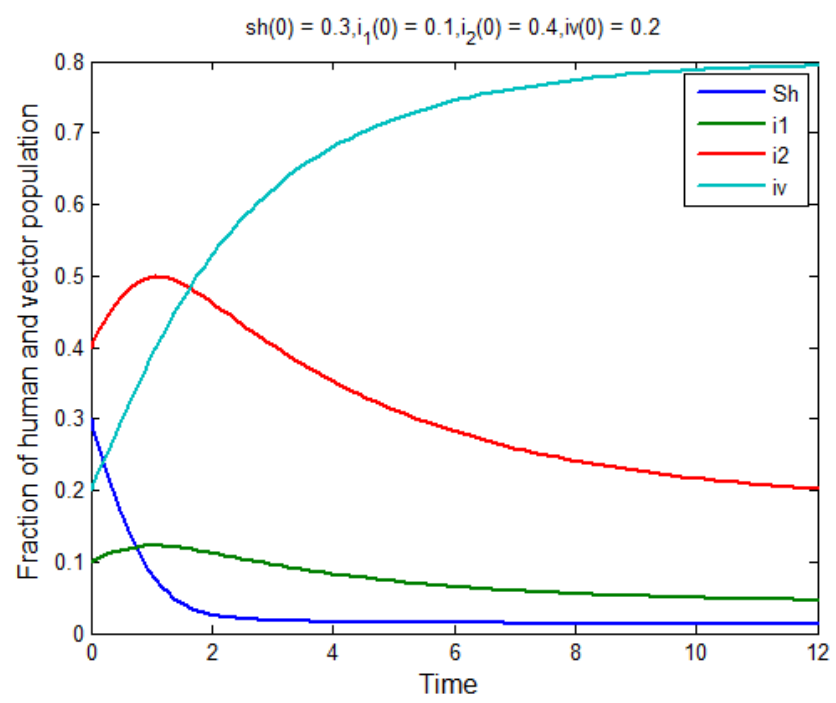

Figure 4. Numerical simulation of zika virus with $\theta=0.1333,=0.2, \alpha=$ $0.225, P_{1}=0.1, P_{2}=0.1$.

In figure 4 the fraction of symptomatic and asymptomatic infected human populations are increase initially and decrease after a certain time because of $P_{1}, P_{2}$ and the values of the interaction rates $\theta$ and. Here the fraction of susceptible human population is decreases with increase time because the interaction coefficients of symptomatic and asymptomatic infected human populations. In all figures the value of $\alpha$ plays a crucial role on the dynamic and spread of zika virus.

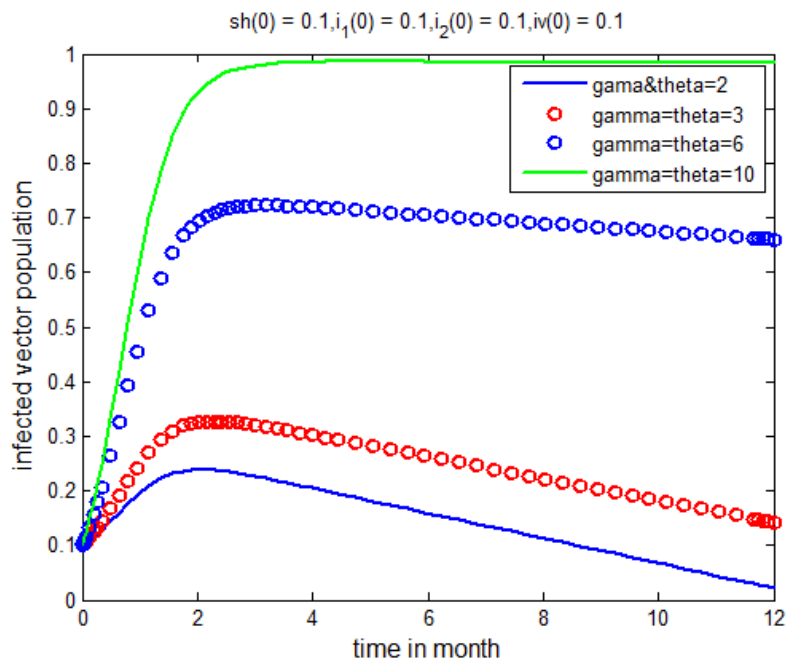

Figure 5. The effect of contact rates from infected human to vector on infected vector population.

In this figure as the contact rates from symptomatic and asymptomatic infected human population to susceptible vector increases proportionally infected vector populations increase rapidly. 


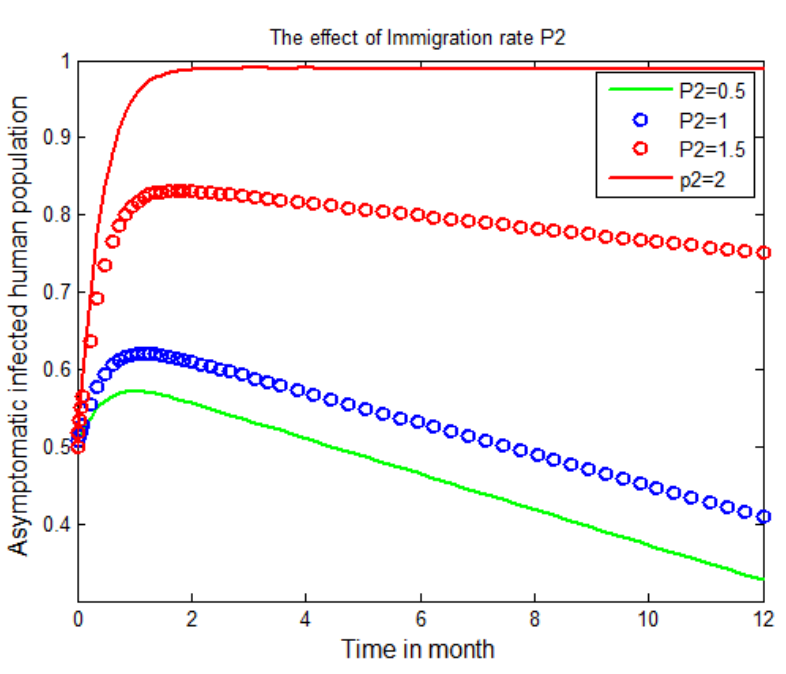

Figure 6. The effect of asymptomatic immigrants on infected human population.

In figure (6) as the rate of asymptomatic infected immigrants increase the infected human population becomes increase and this leads to increase the chance on infection for susceptible vector from those infected humans.

In our model, the interaction coefficient $\theta$ from symptomatic infective to susceptible vector, $Y$ from asymptomatic infected human to susceptible vector, $\Phi$ from infected vector to susceptible human are more sensitive parameters. $P_{1}$ the symptomatic immigration rate, $P_{2}$ asymptomatic immigration rates and $\alpha$ the transmission probability from infected vector to susceptible human also sensitive parameters.

In general, we could observe that increasing the contact rates, human to mosquito and mosquito to human, leads to the outbreak of the disease and results an increasing zika virus prevalence. However, controlling these parameters with different control strategies allow the reproduction number to become less than 1 , and then the disease dies out.

\section{Conclusions}

Intervention measures, to prevent or reduce the transmission of vector born, are currently being used with a degree of success in some parts of the world. Some of the methods used include house spraying with residual insecticides and most recently the use of mosquito bed nets. These methods operate by reducing the contact rates between the mosquitoes and humans. Spraying reduces mosquito longevity (and perhaps also fertility). This strategy is also likely to kill mosquitoes so it would increase the chances of killing infected mosquitoes and increase the mosquito death rate $\mu_{v}$.. Preventing mosquitohuman contacts should reduce the number of bites per mosquito. Controlling the symptomatic and asymptomatic infected immigrants also reduce the contact rate between the infected host and susceptible vector.

In this study, we have derived and analyzed a mathematical model in order to understand the transmission and spread of the zika virus disease. The model is modified by eliminating the recovery human and susceptible mosquito population from the system and by considering symptomatic and asymptomatic infective immigrants. We defined a reproductive number $R_{0}$ which provides the expected number of new infections from one infectious individual over the duration of the infectious period given that all other members of the population are susceptible. We showed the existence and stabilities of equilibrium points of the model. In the model, we demonstrated that the disease-free equilibrium point $E_{0}$ is stable is $R_{0}<1$, and unstable if $R_{0}>1$.

Numerical simulation of the model shows the dynamic properties of human and vector compartments versus time and the stabilities of the equilibrium points. One can observe from the simulations that the infected human population increases with larger values of the contact rates from mosquito to human population and human to mosquito population and with rates of infective immigrants. We notice that in order to reduce the basic reproduction number below 1 , focus on reduction of the contact between mosquito vector and human host and controlling infective immigrants. Thus, using treated bed nets, and insecticides that would reduce the mosquito population and keep the human population stable.

\section{References}

[1] Ebeneza. B, Kazem Oake. O. 2016. Mathematical modeling of Zika virus. Asian Pacific Journal of Tropical Disease. Sep 2016.

[2] Raúl Isea, Karl E. Lonngren. A Preliminary Mathematical Model for the dynamic Transmission of Dengue, Chikungunya and Zika. American Journal of Modern Physics and Application. 24 June, 2016.

[3] Victor M., Baltazar E., Derdei B., Susan A. Holechek, and Carlos C. Simon. Role of short-term dispersal on the dynamics of Zika virus. Center for Infectious Diseases and Vaccinology, the Biodesign Institute, Arizona State University 16 Mar 2016.

[4] Nidhi Nirwani, V. H. Badshah, R. Khandelwal. A Mathematical Model of Malaria Disease with Vertical Transmission. Published by Canadian Center of Science and Education. 7 August 2015.

[5] Mary K. K., Tomas Allen, Veronika Frank, Ravi S. Santhana, Christopher D. The origin and spread of a mosquito-borne virus. Bulletin of the World Health Organization. 9 February 2016.

[6] T. Alex Perkins, Amir S S., Corrine W. R., Moritz U. G. Kraemer, Andrew J. Tatem. Model based projections of Zika virus infections in childbearing women in the America. 12 Feb.2016 http://dx.doi.org/10.1101/039610.

[7] Maimuna S M., Emily C., Durland F., John S. Estimating a feasible serial interval range for Zika fever. Bulletin of the World Health Organization. 9 February 2016.

[8] Abadi Abay Gebremeskel, Harald Elias Krogstad. Mathematical Modeling of Endemic Malaria Transmission. American Journal of Applied Mathematics. 12 February 2015.

[9] Mikayla C. Chubb Kathryn H. J. Mathematical modeling and the epidemiological research. European Journal of Epidemiology. 27 October 2009. 
[10] S. Olaniyi, O. S. Obabiyi. Mathematical model for malaria transmission dynamics in human and Mosquito populations with non-linear force of infection. International Journal of Pure and Applied Mathematics. 15 August 2013.

[11] Syafruddin Side, Salmi Md Noorani. A SIR Model for Spread of Dengue Fever Disease. World Journal of Modeling and Simulation. 14 April 2013.

[12] Zika virus fact sheet. Ethiopian midwives association.

[13] Epidemiological alert. Neurological syndrome, congenital malformations, and Zika virus infection. Implications for public health in the Americas. Pan American Health Organization (PAHO) / World Health Organization (WHO). 1 December 2015.

[14] Katherine F. D., Alexa O., Emily P. Interim Zika Virus Clinical Guidance and Recommendations. Centers for Disease Control and Prevention. 26 January, 2016.

[15] Rapid Risk Assesment, Zika virus disease epidemic: potential association with microcephaly and Guillain-Barré syndrome. 20 January 2016.

[16] Thais D. S, Wanderson K. D. Zika Virus and the Guillain-
Barre Syndrome: Case Series from Seven Countries. The new England journal of medicine. 20 October 2016.

[17] Sandip M., Ram R. S, and Somdatta S. Mathematical models of malaria. Mandal et al. Malaria Journal (2011, 10: 202).

[18] Kelly A. T. Benign bacteria block mosquitoes from transmitting Zika, chikungunya viruses. University of Wisconsin U Madison. 1 Jun 2016.

[19] Tadele Tesfa Tegegne, Purnachandra Rao Koya, Temesgen Tibebu Mekonnen. Impact of Heterosexuality and Homosexuality on the transmission and dynamics of HIV/AIDS. IOSR Journal of Mathematics. Dec. 2016. eISSN: 2278 - 5728, p-ISSN: 2319 - 765X. Volume 12, Issue 6 Ver. V (Nov. - Dec.2016), PP 38-49 www.iosrjournals.org.

[20] Iurii Bakach. A survey of mathematical models of Dengue fever. Georgia Southern University, electronic thesis and dissertation.

[21] Yingyun Shen. Mathematical Models of Dengue Fever and Measures to Control It. Florida State University Libraries, Electronic Theses, Treatises and Dissertations. Semester, 2014. 